\title{
RESPIRE 1: a phase III placebo-controlled randomised trial of ciprofloxacin dry powder for inhalation in non-cystic fibrosis bronchiectasis
}

\author{
Anthony De Soyza $\mathbb{1}^{1}$, Timothy Aksamit ${ }^{2}$, Tiemo-Joerg Bandel ${ }^{3}$, \\ Margarita Criollo ${ }^{4}$, J. Stuart Elborn ${ }^{5}$, Elisabeth Operschall ${ }^{6}$, Eva Polverino ${ }^{7}$, \\ Katrin Roth ${ }^{6}$, Kevin L. Winthrop ${ }^{8}$ and Robert Wilson ${ }^{9}$
}

Affiliations: ${ }^{1}$ Newcastle University and Freeman Hospital, Newcastle upon Tyne, UK. ${ }^{2}$ Mayo Clinic, Rochester, MN, USA. ${ }^{3}$ Bayer AG, Wuppertal, Germany. ${ }^{4}$ Bayer Inc., Mississauga, ON, Canada. ${ }^{5}$ National Heart and Lung Institute, Imperial College London and Royal Brompton Hospital, London, UK. ${ }^{6}$ Bayer AG, Berlin, Germany. ${ }^{7}$ Hospital Universitari Vall d'Hebron, Institut de Recerca Vall d'Hebron, Barcelona, Spain. ${ }^{8}$ Oregon Health and Science University, Portland, OR, USA. ${ }^{9}$ Royal Brompton Hospital and Imperial College London, London, UK.

Correspondence: Anthony De Soyza, Institute of Cellular Medicine, Newcastle University and Freeman Hospital, Framlington Place, Newcastle upon Tyne, NE2 4HH, UK. E-mail: anthony.de-soyza@newcastle.ac.uk

@ERSpublications

In RESPIRE 1, 14-day on/off cycles of ciprofloxacin dry powder for inhalation reduced bronchiectasis exacerbations http://ow.ly/po4s30gNfl0

Cite this article as: De Soyza A, Aksamit T, Bandel T-J, et al. RESPIRE 1: a phase III placebo-controlled randomised trial of ciprofloxacin dry powder for inhalation in non-cystic fibrosis bronchiectasis. Eur Respir J 2018; 51: 1702052 [https://doi.org/10.1183/13993003.02052-2017].

ABSTRACT We evaluated the efficacy and safety of ciprofloxacin dry powder for inhalation (DPI) in patients with non-cystic fibrosis bronchiectasis, two or more exacerbations in the previous year and predefined bacteria in sputum.

In this phase III, double-blind, placebo-controlled trial, patients were randomised 2:1 to twice-daily ciprofloxacin DPI $32.5 \mathrm{mg}$ or placebo in two treatment regimens consisting of on/off treatment cycles of 14 or 28 days for 48 weeks. The primary end-points were time to first exacerbation and frequency of exacerbations.

A total of 416 patients were randomised to the 14-day on/off regimen (ciprofloxacin DPI $(n=137)$ and placebo $(n=68)$ ) or the 28-day on/off regimen (ciprofloxacin DPI $(n=141)$ and placebo $(n=70)$ ). Ciprofloxacin DPI 14 days on/off significantly prolonged time to first exacerbation versus pooled placebo (median time $>336$ versus 186 days; hazard ratio $0.53,97.5 \%$ CI $0.36-0.80 ; \mathrm{p}=0.0005)$ and reduced the frequency of exacerbations compared with matching placebo by $39 \%$ (mean number of exacerbations 0.6 versus 1.0; incidence rate ratio 0.61, 97.5\% CI 0.40-0.91; $\mathrm{p}=0.0061$ ). Outcomes for ciprofloxacin DPI 28 days on/off were not statistically significantly different from placebo. The safety profile of ciprofloxacin DPI was favourable.

Ciprofloxacin DPI was well tolerated and has the potential to be an effective treatment option in noncystic fibrosis bronchiectasis.

This article has supplementary material available from erj.ersjournals.com

Received: Oct 062017 | Accepted after revision: Nov 202017

This study is registered at ClinicalTrials.gov with identifier NCT01764841.

Support statement: This study was supported by Bayer AG. Funding information for this article has been deposited with the Crossref Funder Registry.

Conflict of interest: Disclosures can be found alongside this article at erj.ersjournals.com

Copyright OERS 2018 


\section{Introduction}

Bronchiectasis is a chronic respiratory disease characterised by reduced quality of life and repeated exacerbations that are caused by airway infection and inflammation [1,2]. Exacerbations are associated with an increased symptom burden; morbidity and mortality risks also both increase with exacerbation frequency [2]. Exacerbations are more common in patients with chronic bacterial infection. Pseudomonas aeruginosa in particular is a risk factor for mortality and hospitalisation [2]. The major management goals for non-cystic fibrosis bronchiectasis (NCFB) are to reduce exacerbations and prevent disease progression [3-5], but there are currently no therapies approved for these purposes.

Ciprofloxacin dry powder for inhalation (DPI) is in development to reduce exacerbations in patients with NCFB with significant disease burden. It is an antibiotic drug-device combination comprising ciprofloxacin inhalation powder, formulated using PulmoSphere technology (Novartis, San Carlos, CA, USA) [6], and a breath-actuated pocket-sized inhaler (supplementary figure S1). Ciprofloxacin DPI achieves high and reproducible levels of drug deposition across ventilated lung areas in patients with NCFB [7]. The 28-day cyclical regimen has traditionally been used in cystic fibrosis (CF) with the intention of maximising treatment effects while minimising resistance, but the appropriateness of this treatment strategy for NCFB is unknown. A phase II study of ciprofloxacin DPI $32.5 \mathrm{mg}$ twice daily for 28 days in patients with NCFB showed a $>3$-fold log reduction in total bacterial load in sputum; the most common pathogens were P. aeruginosa, Haemophilus influenzae and Staphylococcus aureus. Notably, the greatest reductions in bacterial load occurred early during treatment $(8 \pm 1$ days) and persisted during therapy; bacterial load increased soon after treatment stopped, returning to almost baseline within 2 months of end of treatment (EOT) [8]. These findings suggested that a shorter treatment cycle, such as 14 days on/off, may be appropriate for ciprofloxacin DPI treatment in patients with NCFB.

The aim of the two phase III RESPIRE trials for ciprofloxacin DPI was to evaluate the efficacy and safety of 14- and 28-day on/off ciprofloxacin DPI in prolonging time to first exacerbation and reducing the frequency of exacerbations in patients with NCFB over 48 weeks [9]. Here, we report here the results of the first study, RESPIRE 1 (ClinicalTrials.gov identifier NCT01764841).

\section{Methods}

\section{Study design and treatment}

Full details of the study design have been published [9]. In brief, RESPIRE 1 was a parallel-group, randomised, double-blinded, international, placebo-controlled trial in which patients were randomised 2:1 (ciprofloxacin DPI:placebo) by a computerised randomisation program to receive intermittent regimens of twice-daily ciprofloxacin DPI $32.5 \mathrm{mg}$ or placebo. Treatment was administered in cycles of 14 days on/off (12 cycles) or 28 days on/off ( 6 cycles) for 48 weeks. Patients were followed for 8 weeks after the last dose (figure 1). Comparisons were made between active treatment groups and matching placebo (i.e. patients randomised to 14- or 28-day on/off placebo versus the comparable ciprofloxacin DPI regimen) or pooled placebo (all patients treated with placebo versus the stated ciprofloxacin DPI regimen) depending on the

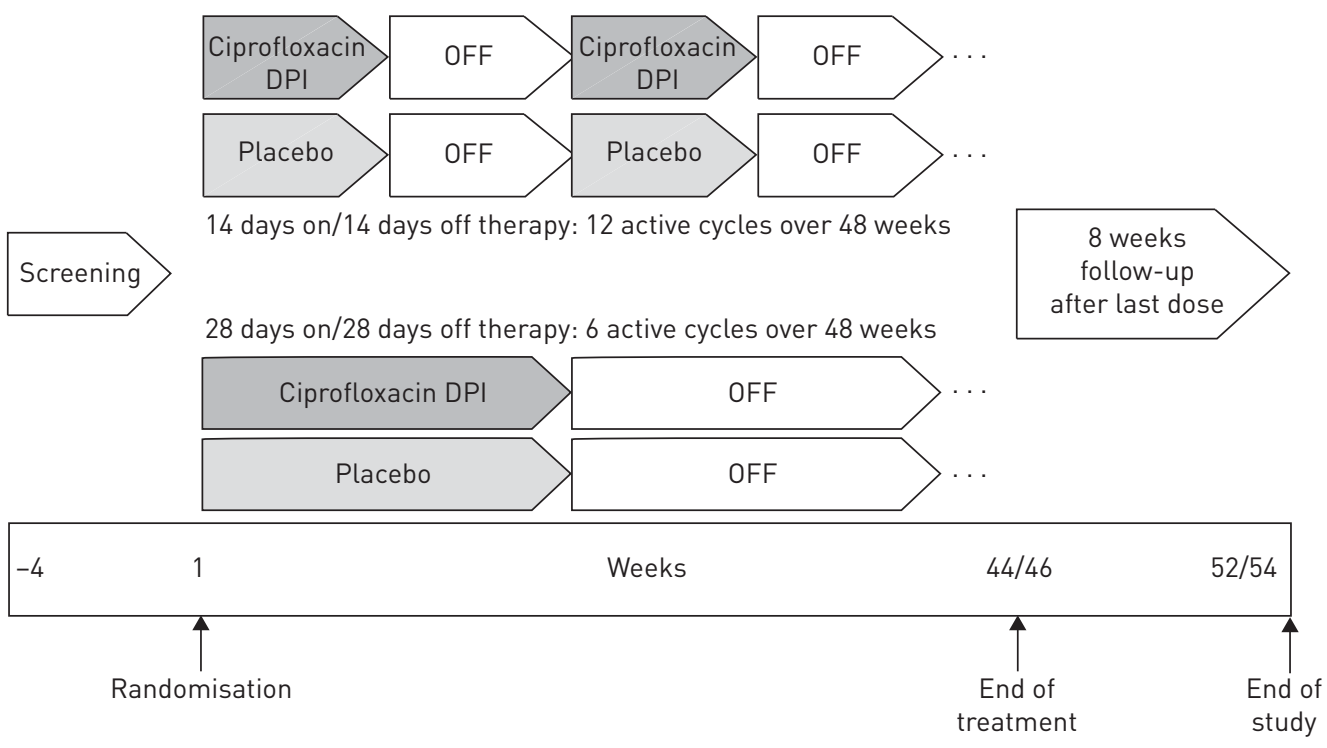

FIGURE 1 RESPIRE study design. DPI: dry powder for inhalation. 
specific outcome (see the End-points section). Compliance was assessed via collection of study medication kits at each visit and was based on days on-treatment. Off-cycles or treatment interruptions, e.g. due to exacerbations, were not included in calculations of compliance.

All appropriate ethics approvals and patients' written informed consent were obtained.

\section{Study population}

Patients with a primary diagnosis of NCFB (idiopathic or post-infectious aetiology as determined by the investigator), confirmed by computed tomography scan, with stable disease (no symptoms of an exacerbation within the past 30 days) were enrolled [9]. Key inclusion criteria were: at least two exacerbations in the previous 12 months documented in the medical records and confirmed by review of medical records, and a positive culture from a sputum sample at screening for at least one pre-specified pathogen: P. aeruginosa, H. influenzae, Moraxella catarrhalis, S. aureus, Streptococcus pneumoniae, Stenotrophomonas maltophilia or Burkholderia cepacia. Stable chronic macrolide treatment was allowed if used for at least 6 months prior to screening. Patients were excluded if they had received systemic or inhaled antibiotic treatments within 4 weeks prior to study drug administration. Patients with active allergic bronchopulmonary aspergillosis, active or actively treated nontuberculous mycobacterial lung infection or tuberculosis at screening, a primary diagnosis of chronic obstructive pulmonary disease as determined by the clinician, or documented chronic asthma were also excluded.

\section{End-points}

Hierarchical analysis approaches were agreed with regulatory authorities. These differed between the US Food and Drug Administration (FDA) and the European Medicines Agency (EMA)/other agencies (supplementary figure S2). The primary end-points were: 1) time to first exacerbation within 48 weeks after start of treatment for ciprofloxacin DPI versus pooled placebo (FDA) and 2) frequency of exacerbations during the 48-week study for ciprofloxacin DPI versus matching placebo (EMA/others). For the primary end-points, exacerbations were required to meet three criteria: 1) worsening of at least three signs or symptoms (dyspnoea, wheezing, cough, 24-h sputum volume or sputum purulence) beyond normal day-to-day variation for at least 2 consecutive days, 2) fever (body temperature $>38.0^{\circ} \mathrm{C}$ ) or malaise/fatigue and 3) systemic antibiotic treatment ("primary end-point definition"). A less stringent definition of exacerbation (respiratory event with worsening of at least one of the aforementioned signs or symptoms and systemic antibiotic use) was evaluated as a secondary end-point. Additional secondary end-points included microbiological outcomes, quality of life assessments and lung function. The supplementary material includes details of these and the pre-defined subgroups for the primary and secondary end-points (supplementary section S1), and the safety end-points, including adverse events (supplementary section S2).

\section{Analyses}

Details of the randomisation process and sample size calculations have been published previously [9]. The sample size calculations described by Акsаміт et al. [9] reflect an adjustment in sample size based on the low number of exacerbation events in blinded data.

Primary efficacy analyses were conducted on the full analysis set, defined as all randomised patients (identical to the intent-to-treat population). For the primary end-point of time to first exacerbation, defined as time from randomisation until the first qualifying exacerbation, a Cox proportional hazards model was used to test for differences between ciprofloxacin DPI and pooled placebo. The independent variables were treatment group, geographical region, pre-therapy positive culture for $P$. aeruginosa and chronic macrolide use. For the primary end-point of frequency of exacerbations, a Poisson regression model with adjustment for over-/underdispersion, the aforementioned independent variables and time in study as an offset variable was used to analyse the number of exacerbations over 48 weeks compared with matching placebo.

Statistical analyses were conducted in a pre-defined hierarchical order (supplementary figure S2) using SAS version 9.2 (SAS Institute, Cary, NC, USA). If the hypothesis of superiority of ciprofloxacin DPI could not be demonstrated, all subsequent end-points were descriptive only, irrespective of the resulting p-value. To account for multiple testing for two active treatment arms (ciprofloxacin DPI 14 and 28 days on/off), a Bonferroni adjustment was applied, giving an $\alpha$ level of 0.025 (two-sided) for each test.

\section{Results}

Patients

The first patient visit was in May 2013 and the last visit was in March 2016. 416 patients were randomised and $80.3 \%$ had data available at the end-of-study (EOS) visit (figure 2). Individual countries randomising the most patients were Israel (12.7\%), Australia (12.5\%) and New Zealand (12.3\%) (supplementary table S1). 


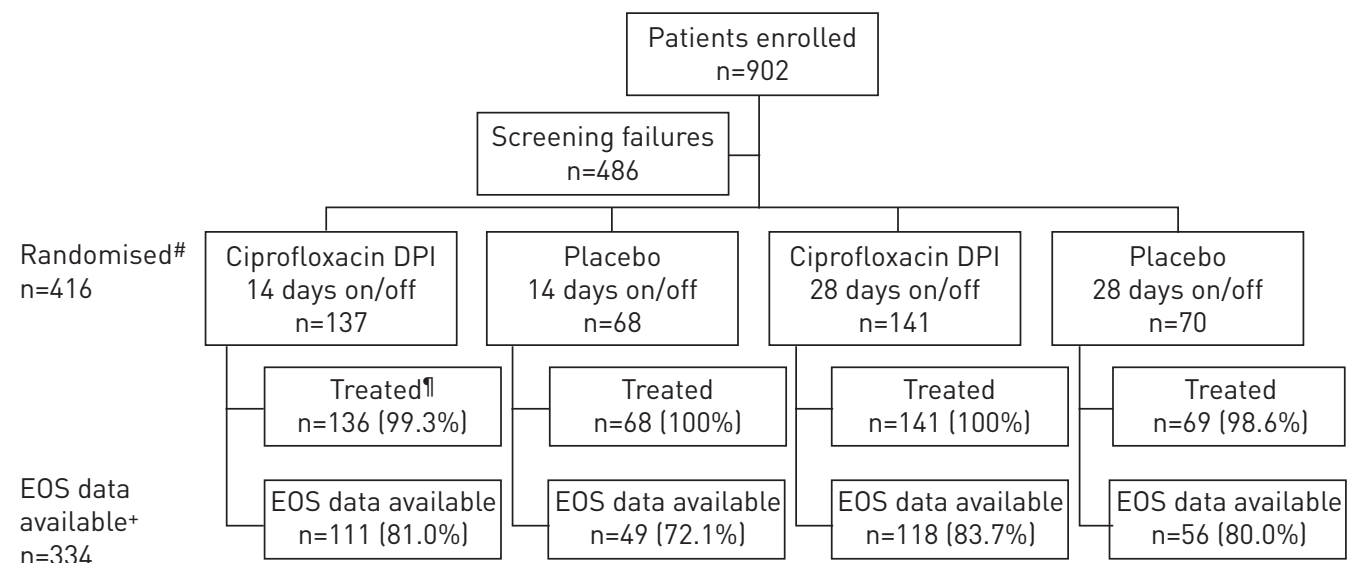

FIGURE 2 Patient disposition. DPI: dry powder for inhalation; EOS: end of study. \#: randomisation stratified by geographical region, pre-therapy positive culture for Pseudomonas aeruginosa and chronic macrolide use; Iः: patients randomised who received at least one treatment dose; ${ }^{+}$: patients with EOS data available include those who completed treatment and subsequent follow-up, or prematurely discontinued treatment but were followed further until the planned EOS (52/54 weeks), or prematurely discontinued treatment but completed the planned 8 weeks of follow-up.

Patient demographics and baseline characteristics were balanced (table 1). Most patients were female ( $68.5 \%$ overall), with a mean age of $\sim 65$ years and $P$. aeruginosa at baseline (61\%) (see supplementary table S2 for other baseline pathogens). Baseline medication use was high and $>80 \%$ of patients received at least one concomitant respiratory medicine (supplementary table S3).

Over $70 \%$ of patients (297 out of 416) completed treatment; the main reasons for early discontinuation were adverse events or withdrawal from the study. Treatment compliance was high $(94 \%$ in active treatment arms) (table 2).

\section{Primary end-points}

Ciprofloxacin DPI 14 days on/off significantly delayed time to first exacerbation compared with pooled placebo over 48 weeks (hazard ratio (HR) 0.53 , 97.5\% CI 0.36-0.80; $\mathrm{p}=0.0005$ ) (table 3 and figure $3 \mathrm{a}$ ). The median time to first exacerbation was $>336$ days (97.5\% CI 290 to $>336$ ) for ciprofloxacin DPI 14 days on/ off and 186 days (97.5\% CI 136-282) for pooled placebo. A delay to first exacerbation was also seen for ciprofloxacin DPI 28 days on/off, but differences did not reach statistical significance versus pooled placebo (HR 0.73, 97.5\% CI 0.50-1.07; $\mathrm{p}=0.0650$; median time to first exacerbation 336 days, $97.5 \%$ CI 206 to $>336$ versus 186 days, 97.5\% CI 136-282) (table 3 and figure 3b).

Ciprofloxacin DPI 14 days on/off significantly reduced frequency of exacerbations versus matching placebo by $39 \%$ over 48 weeks (incidence rate ratio (IRR) $0.61,97.5 \%$ CI $0.40-0.91 ; p=0.0061$ ) (table 3 and figure $4 \mathrm{a})$. The mean \pm SD number of exacerbations during the 48 weeks in the ciprofloxacin DPI 14-day on/ off arm was $0.6 \pm 1.0$ compared with $1.0 \pm 1.1$ in the matching placebo arm. The treatment effect of ciprofloxacin DPI 28 days on/off was lower and not statistically different from matching placebo (table 3 and figure $4 \mathrm{~b}$ ). The mean \pm SD number of exacerbations was $0.8 \pm 1.1$ for ciprofloxacin DPI 28 days on/off and $0.8 \pm 1.0$ for matching placebo.

Over 48 weeks, $61.3 \%$ of patients treated with ciprofloxacin DPI 14 days on/off compared with $52.5 \%$ of patients treated with ciprofloxacin DPI 28 days on/off and $42.8 \%$ of patients in the pooled placebo arm did not experience a protocol-defined exacerbation for the primary end-point.

\section{Subgroup analyses}

For both primary end-points, exploratory analyses showed a consistent positive treatment effect for ciprofloxacin DPI 14 days on/off in all subgroups versus placebo (supplementary figure S3a). Ciprofloxacin DPI 28 days on/off showed similar, but less pronounced, patterns (supplementary figure S3b). In both regimens, the $97.5 \%$ confidence intervals within each subgroup pairing widely overlapped, indicating no effect modification by key pre-specified subgroups.

\section{Secondary end-points}

In the FDA-specified analyses, ciprofloxacin DPI 14 days on/off narrowly missed the first secondary end-point of frequency of exacerbations according to the primary end-point definition (figure 5). The 
TABLE 1 Patient demographics and baseline characteristics

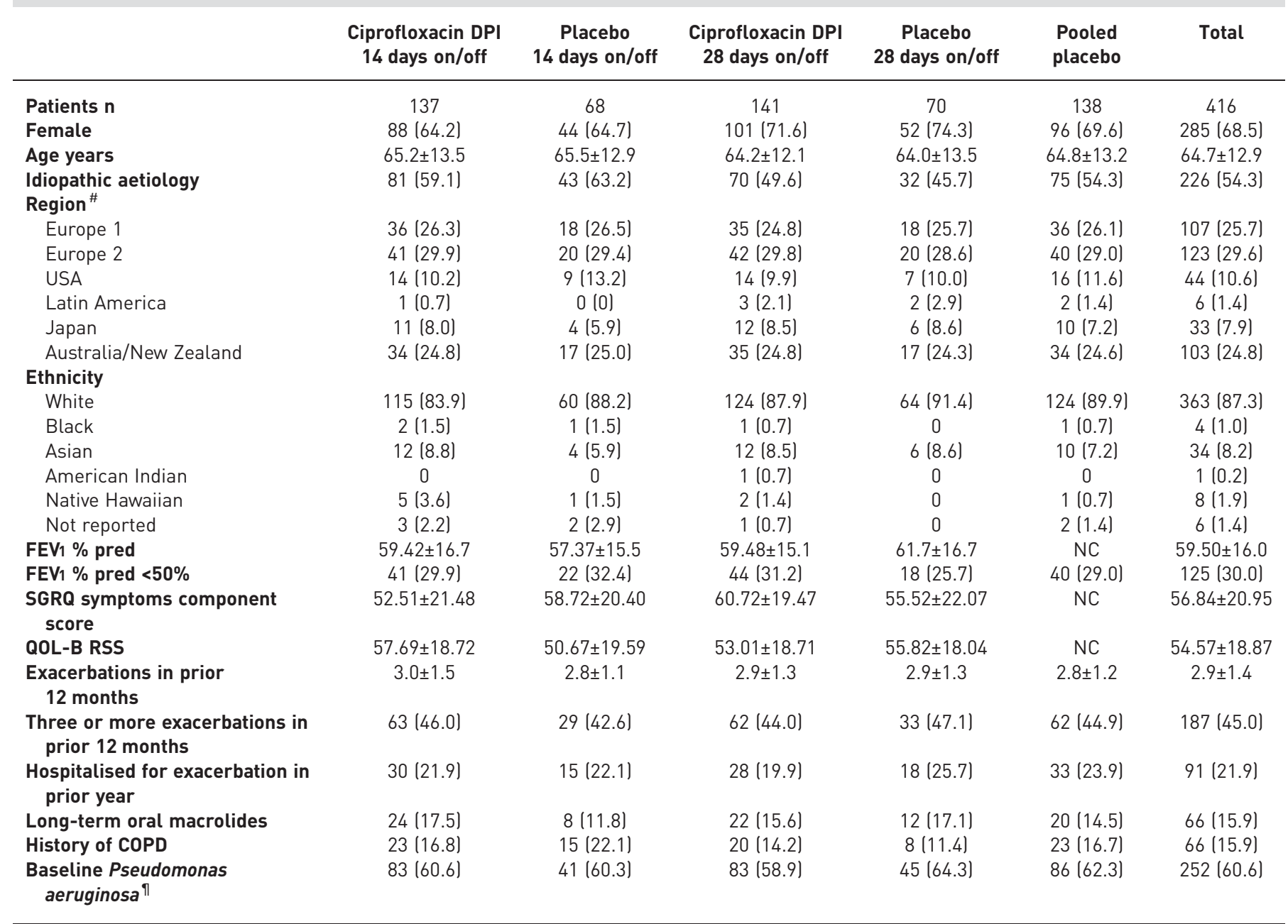

Data are presented as $\mathrm{n}(\%)$ or mean $\pm \mathrm{SD}$, unless otherwise stated. DPI: dry powder for inhalation; FEV1: forced expiratory volume in $1 \mathrm{~s}$; SGRQ: St George's Respiratory Questionnaire; QOL-B RSS: Quality of Life-Bronchiectasis respiratory symptoms domain score; COPD: chronic obstructive pulmonary disease; NC: not calculated. \#: Europe 1: Denmark, France, Germany, Latvia, Slovakia, UK; Europe 2: Israel, Italy, Spain; Latin America: Argentina. ๆ : patients could have more than one species isolated.

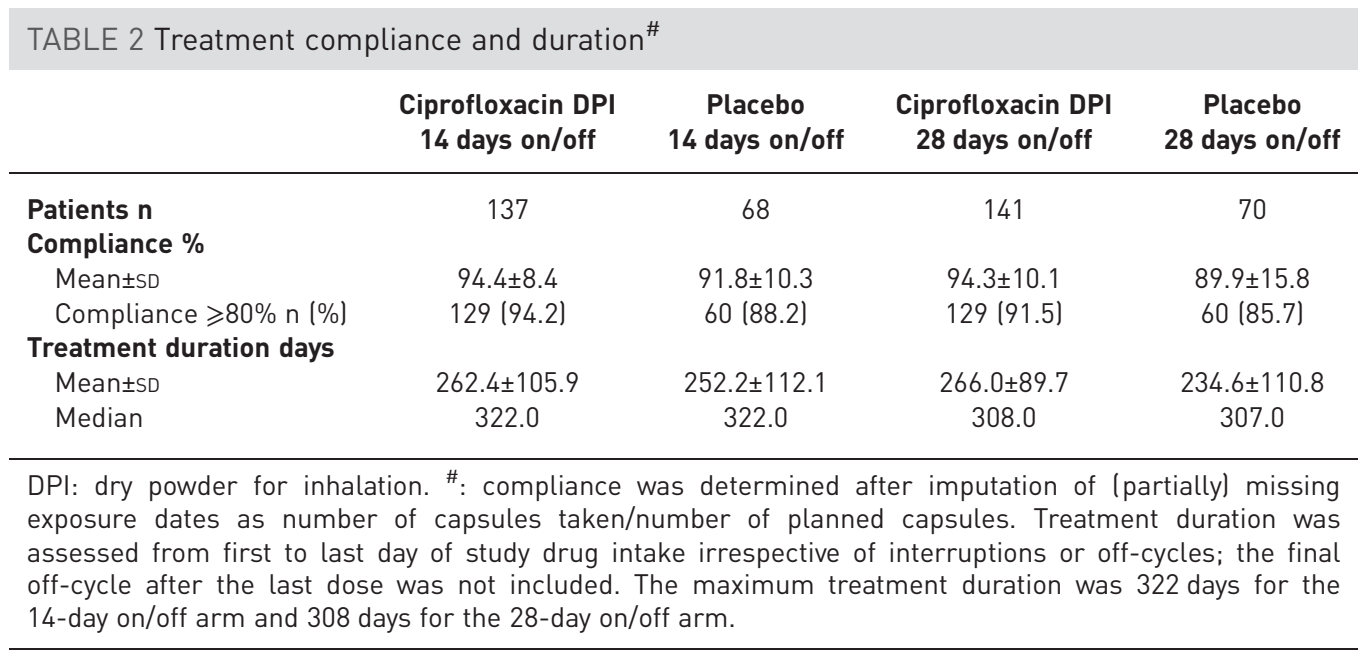


TABLE 3 Primary end-points: time to first exacerbation and frequency of exacerbations ${ }^{\#}$ in patients treated with ciprofloxacin dry powder for inhalation (DPI) versus placebo over 48 weeks

HR or IRR

(97.5\% Cl) p-value (Wald-type test)

\section{Time to first exacerbation}

Ciprofloxacin DPI 14 days on/off versus pooled placebo

Ciprofloxacin DPI 28 days on/off versus pooled placebo

$0.53(0.36-0.80)$

$0.73(0.50-1.07)$

$0.61(0.40-0.91)$

$0.98(0.64-1.48)$
0.0005

0.0650

0.0061

0.8946

Ciprofloxacin DPI 14 days on/off versus matching placebo

Ciprofloxacin DPI 28 days on/off versus matching placebo

HR: hazard ratio; IRR: incidence rate ratio. ${ }^{\#}$ : for the primary end-points, an exacerbation (stringent definition) was required to meet three criteria: 1) worsening in at least three signs or symptoms (dyspnoea, wheezing, cough, 24-h sputum volume or sputum purulencel beyond normal day-to-day variation for at least 2 consecutive days, 2) fever (body temperature $>38.0^{\circ} \mathrm{C}$ ) or malaise/fatigue, and 3) systemic antibiotic treatment; ": HRs were calculated for time to first exacerbation; IRRs were calculated for frequency of exacerbations.

corresponding mean \pm SD number of exacerbations was $0.9 \pm 1.2$ in the ciprofloxacin DPI 14-day on/off arm and 1.2 \pm 1.3 for the pooled placebo groups. Sensitivity analyses (number of exacerbation events as defined for the primary end-point with time in study as an offset in the model for patients who did not complete the 48-week study, rather than extrapolation of the number of events) indicated a positive nominally significant effect in the ciprofloxacin DPI 14-day on/off arm (IRR 0.6898, 97.5\% CI 0.4833-0.9845; $\mathrm{p}=0.0193$ ). As expected, the mean \pm SD number of exacerbations according to the less stringent definition was higher (ciprofloxacin DPI 14 days on/off $1.2 \pm 1.5$ versus pooled placebo $1.6 \pm 1.5$ ).

Due to the hierarchical nature of the analysis, which was determined a priori, formal confirmatory significance testing was not performed for any further secondary end-points in the FDA analysis plan.

For the EMA/others analysis, ciprofloxacin DPI 14 days on/off reached the first secondary end-point (time to first exacerbation) (figure 5a). The second secondary end-point (frequency of exacerbations according to a less stringent definition) was consistent with the primary end-point and a significant reduction was seen in the mean \pm SD number of exacerbations according to the less stringent definition for ciprofloxacin DPI 14 days on/off $(0.9 \pm 1.3)$ versus matching placebo (1.4 \pm 1.3$)$. Ciprofloxacin DPI 14 days on/off was also statistically significantly different versus placebo for the next two secondary end-points (eradication of pre-specified baseline pathogens at EOT versus pooled placebo and change from baseline to EOT in St George's Respiratory Questionnaire (SGRQ) symptoms component score versus pooled placebo).

As ciprofloxacin DPI 28 days on/off did not reach statistical significance for the primary end-point for either the FDA- or EMA/others-specified analysis, secondary end-point analyses were only descriptive; data are shown in figure $5 \mathrm{~b}$.

\section{Safety}

Ciprofloxacin DPI was well tolerated and the incidence of adverse events and treatment-emergent adverse events, including drug-related and serious treatment-emergent adverse events, was similar among groups (table 4 and supplementary table S4). Adverse events occurred in $83 \%$ of patients overall, with no imbalances between treatment groups in the overall adverse event rates. Treatment-emergent respiratory adverse events, including bronchospasm, were comparable across treatment groups. Discontinuations due to treatment-emergent adverse events were similar among groups (table 4).

One case of tendonitis (placebo group) was seen; there were no reported cases of tendon rupture. Musculoskeletal adverse events occurred at a numerically higher rate in the ciprofloxacin DPI groups (approximately $20 \%$ versus $12 \%$ ); this difference was primarily due to higher rates of arthralgia, back pain and osteoarthritis.

Reported serious treatment-emergent adverse events were mostly respiratory related (supplementary table S4). There were no apparent differences in the type or number of serious treatment-emergent adverse events between the active and placebo groups. There were a total of six treatment-emergent deaths reported (one in the ciprofloxacin DPI 14-day on/off arm, two in the ciprofloxacin DPI 28-day on/off arm and three in the pooled placebo group) (supplementary table S4). None of these deaths were considered related to the study drug. 

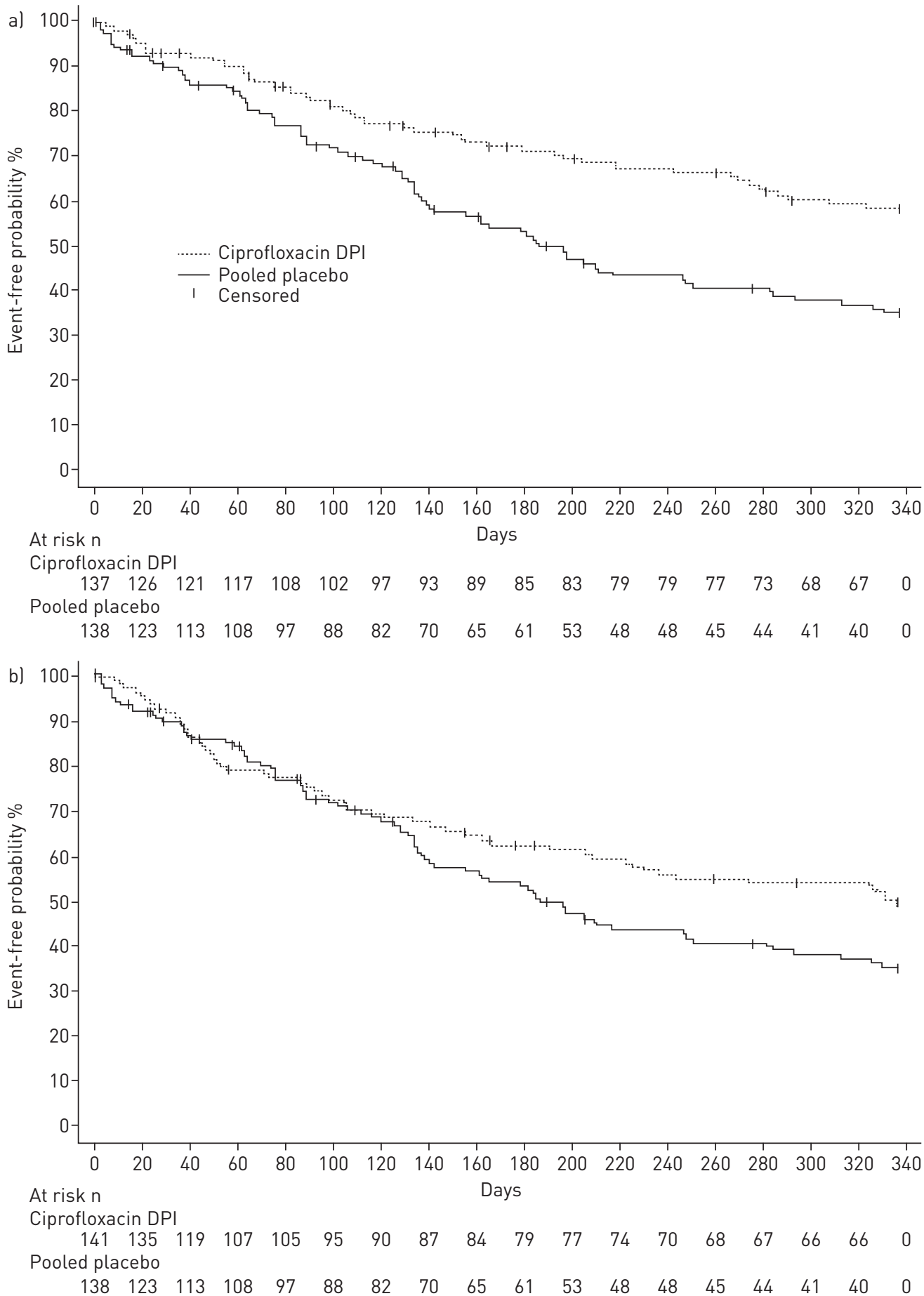

FIGURE 3 Time to first exacerbation for patients receiving ciprofloxacin dry powder for inhalation (DPI) a) 14 days on/off or placebo and b) 28 days on/off or placebo. For the primary end-point exacerbations were required to meet three criteria: 1) worsening in at least three signs or symptoms (dyspnoea, wheezing, cough, 24-h sputum volume or sputum purulencel beyond normal day-to-day variation for at least 2 consecutive days, 2) fever (body temperature $>38.0^{\circ} \mathrm{C}$ ) or malaise/fatigue, and 3) systemic antibiotic treatment.

Changes in ciprofloxacin minimal inhibitory concentrations

At baseline, 102 out of 416 patients (24.5\%) had a pathogen with an elevated minimal inhibitory concentration (MIC) for ciprofloxacin (defined as resistant based on systemic ciprofloxacin breakpoints as specified in supplementary section S2) isolated from their sputum. The number of patients with the development of pathogens with elevated MICs (resistant by systemic breakpoints) from pre-treatment to any time-point during the study was observed more frequently in ciprofloxacin DPI patients compared 
a) 100
Ciprofloxacin DPI

Placebo

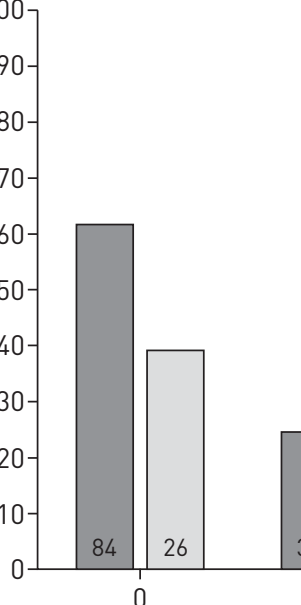

b) 100

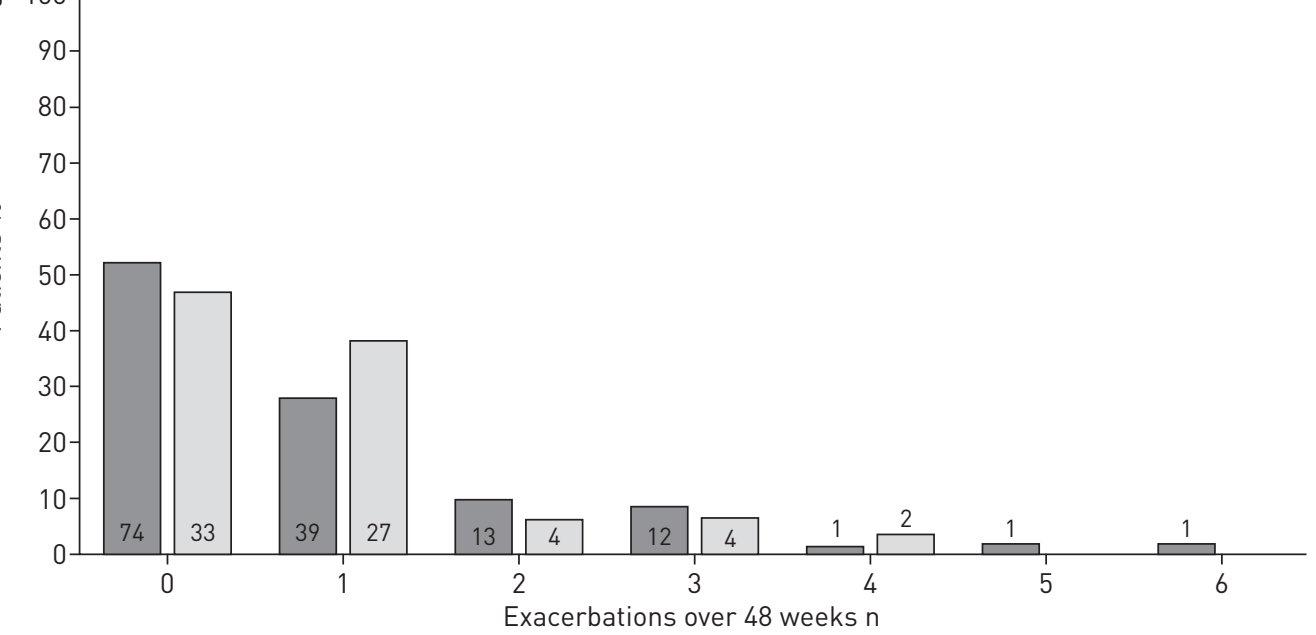

FIGURE 4 Descriptive frequency of exacerbations over 48 weeks for patients receiving ciprofloxacin dry powder for inhalation (DPI) a) 14 days on/off or matching placebo and b) 28 days on/off or matching placebo. Numbers within or above bars indicate number of patients. For the primary end-point exacerbations were required to meet three criteria: 1) worsening in at least three signs or symptoms (dyspnoea, wheezing, cough, 24-h sputum volume or sputum purulence) beyond normal day-to-day variation for at least 2 consecutive days, 2) fever (body temperature $>38.0^{\circ} \mathrm{C}$ ) or malaise/fatigue, and 3) systemic antibiotic treatment.

with placebo (28 out of 137 (20.4\%), 37 out of 141 (26.2\%) and 17 out of 138 (12.3\%) for ciprofloxacin DPI 14 days on/off, ciprofloxacin DPI 28 days on/off and pooled placebo, respectively). At the EOS visit, which occurred 8 weeks after EOT, pathogens with elevated MICs were isolated from 26 patients (6.3\%) with susceptible pathogens at baseline (10 out of $137(7.3 \%), 13$ out of $141(9.2 \%)$ and three out of 138 (2.2\%) for ciprofloxacin DPI 14 days on/off, ciprofloxacin DPI 28 days on/off and pooled placebo, respectively). The percentage of patients with at least one isolate from sputum with an elevated MIC at any time-point including both baseline and EOS as defined by systemic ciprofloxacin breakpoints was $54.0 \%$ for ciprofloxacin DPI 14 days on/off and 53.9\% for ciprofloxacin DPI 28 days on/off versus $36.2 \%$ for the pooled placebo group.

\section{Discussion}

RESPIRE is the largest placebo-controlled, double-blind phase III trial programme in NCFB and the first to show a significant benefit for long-term intermittent inhaled antibiotics in patients with NCFB. RESPIRE 1 is also the first to investigate the efficacy of both 14- and 28-day treatment cycles. Cycles of 28 days are conventionally used in CF treatment [10] and have previously been studied in NCFB, with limited success $[11,12]$. The choice of 14 days on/off as a treatment regimen in the RESPIRE programme was guided by data from the phase II trial [8].

The ciprofloxacin DPI 14-day on/off arm resulted in statistically significant improvements in time to first exacerbation and frequency of exacerbations over 48 weeks. Subgroup analyses suggest that this regimen 


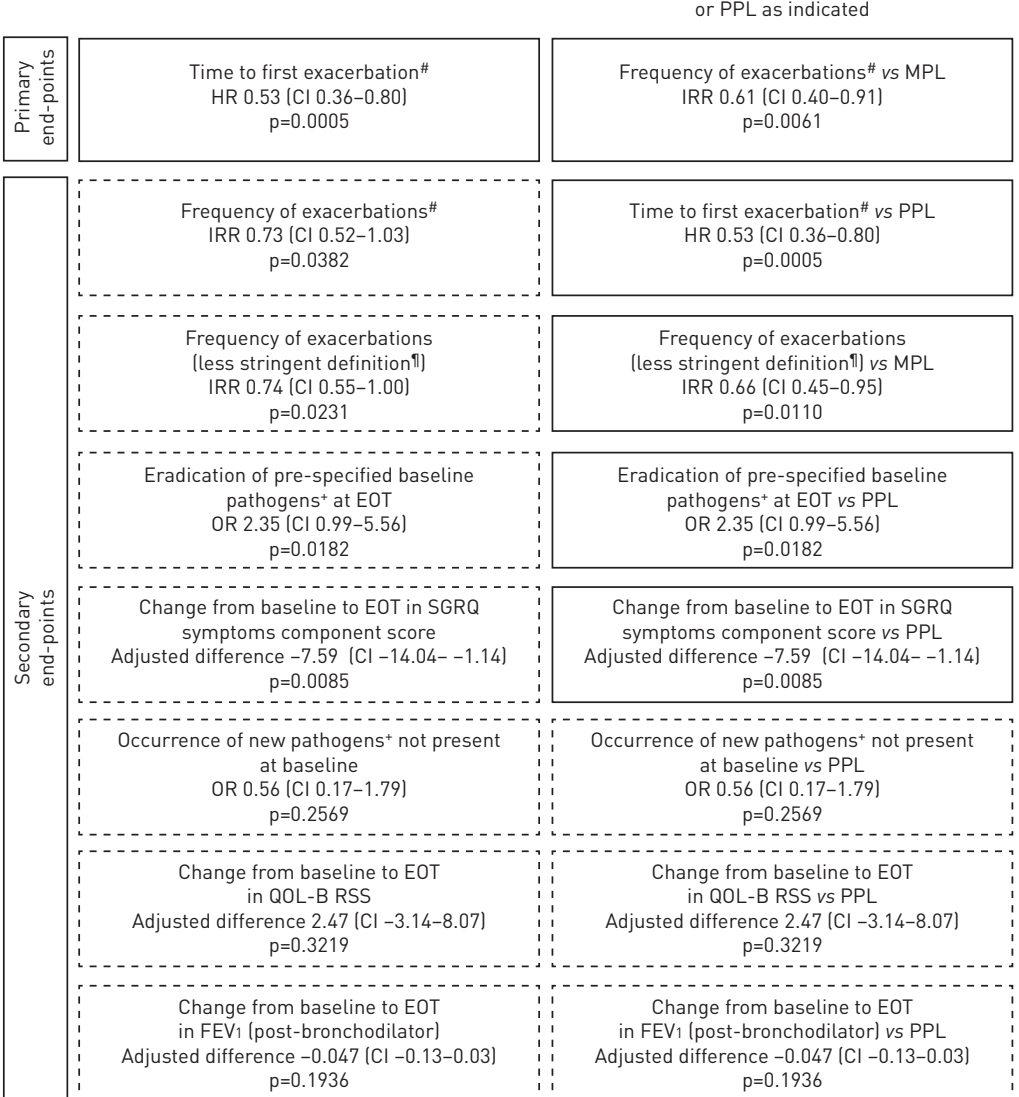

b)

\begin{tabular}{|c|c|c|}
\hline 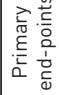 & $\begin{array}{l}\text { Time to first exacerbation\# } \\
\text { HR } 0.73(\mathrm{Cl} 0.50-1.07) \\
\mathrm{p}=0.0650\end{array}$ & 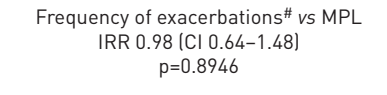 \\
\hline \multirow{7}{*}{ 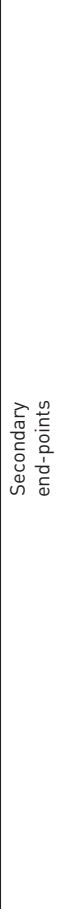 } & $\begin{array}{l}\text { Frequency of exacerbations } \# \\
\text { IRR } 0.86(C l 0.63-1.18) \\
p=0.2944\end{array}$ & $\begin{array}{l}\text { Time to first exacerbation\# vs PPL } \\
\text { HR } 0.73(\mathrm{Cl} 0.50-1.07) \\
\mathrm{p}=0.0650\end{array}$ \\
\hline & $\begin{array}{l}\text { Frequency of exacerbations } \\
\text { (less stringent definition?) } \\
\text { IRR } 0.87 \text { (CI 0.66-1.16) } \\
\text { p } 0.2761\end{array}$ & $\begin{array}{l}\text { Frequency of exacerbations } \\
\text { (less stringent definition?) vs MPL } \\
\text { IRR } 0.97 \text { (CI } 0.67-1.41 \text { ) } \\
\mathrm{p}=0.8628\end{array}$ \\
\hline & 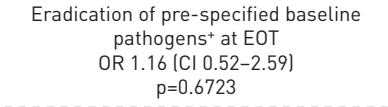 & $\begin{array}{c}\text { Eradication of pre-specified baseline } \\
\text { pathogens } \text { at EOT vs PPL } \\
\text { OR } 1.16(1 \mathrm{Cl} 0.52-2.59] \\
\text { p=0.6723 }\end{array}$ \\
\hline & $\begin{array}{l}\text { Change from baseline to EOT in SGRQ } \\
\text { symptoms component score } \\
\text { Adjusted difference }-5.21 \text { (CI }-11.53-1.10) \\
p=0.0636\end{array}$ & $\begin{array}{l}\text { Change from baseline to EOT in SGRQ } \\
\text { symptoms component score vs PPL } \\
\text { Adjusted difference }-5.21 \text { (CI -11.53-1.10) } \\
p=0.0636\end{array}$ \\
\hline & $\begin{array}{l}\text { Occurrence of new pathogens }{ }^{+} \text {not present } \\
\text { at baseline } \\
\text { OR } 0.36 \text { (C) } 1.0 .10-1.31) \\
p=0.0582\end{array}$ & $\begin{array}{l}\text { Occurrence of new pathogens }{ }^{+} \text {not present } \\
\text { at baseline vs PPL } \\
\text { OR } 0.36 \text { (CI I. } 0.10-1.31) \\
\text { p=0.0582 }\end{array}$ \\
\hline & $\begin{array}{l}\text { Change from baseline to EOT } \\
\text { in QOL-B RSS } \\
\text { Adjusted difference } 1.118 \text { (Cl -4.17-6.53) } \\
p=0.6187\end{array}$ & $\begin{array}{l}\text { Change from baseline to EOT } \\
\text { in QOL-B RSS vs PPL } \\
\text { Adjusted difference } 1.18 \text { (CI }-4.17-6.53) \\
p=0.6187\end{array}$ \\
\hline & $\begin{array}{l}\text { Change from baseline to EOT } \\
\text { in FEV (post-bronchodilator) } \\
\text { Adjusted difference }-0.032(\mathrm{Cl}-0.11-0.05) \\
p=0.3700\end{array}$ & $\begin{array}{l}\text { Change from baseline to EOT } \\
\text { in FEV (post-bronchodilator) vs PPL } \\
\text { Adjusted difference - } 0.032 \text { (CI }-0.11-0.05 \text { ) } \\
p=0.3700\end{array}$ \\
\hline
\end{tabular}

FIGURE 5 Results for ciprofloxacin dry powder for inhalation (DPI) in US Food and Drug Administration (FDA) and European Medicines Agency (EMA)/other agencies hierarchical analyses for ciprofloxacin DPI a) 14 days on/off and b) 28 days on/off versus pooled placebo (PPL) or matching placebo (MPL). HR: hazard ratio; IRR: incidence rate ratio; EOT: end of treatment; SGRQ: St George's Respiratory Questionnaire; QOL-B RSS: Quality of Life-Bronchiectasis respiratory symptoms domain score; FEV1: forced expiratory volume in 1 s. \#: the primary (stringent) definition of exacerbation was a respiratory event that met three separate criteria: 1) worsening in at least three signs or symptoms (dyspnoea, wheezing, cough, 24-h sputum volume or sputum purulencel beyond normal day-to-day variation for at least 2 consecutive days, 2) fever (body temperature $>38.0^{\circ} \mathrm{C}$ ) or malaise/fatigue, and 3 ) systemic antibiotic treatment; ${ }^{9}$ : the less stringent definition of exacerbation was a respiratory event with worsening of at least one of the aforementioned signs or symptoms and systemic antibiotic use; ${ }^{+}:$Pseudomonas less stringent definition of exacerbation was a respiratory event with worsening of at least one of the aforementioned signs or symptoms and systemic antibiotic use; ${ }^{+}$: Pseudomonas
aeruginosa, Haemophilus influenzae, Moraxella catarrhalis, Staphylococcus aureus, Streptococcus pneumoniae, Stenotrophomonas maltophilia or Burkholderia cepacia leradication of pathogens aeruginosa, Haemophilus influenzae, Moraxella catarrhalis, Staphylococcus aureus, Streptococcus pneumoniae, Stenotrophomonas maltophilia or Burkholderia cepacia leradication of pathogens
was defined as a negative culture result at EOT for all pre-specified pathogens in a subject with a positive baseline culture for at least one pre-specified pathogen; occurrence of a new was defined as a negative culture result at EOT for all pre-specified pathogens in a subject with a positive baseline culture for at least one pre-specified pathogen; occurrence of a new pathogen was defined as a positive culture for at least one pre-specified pathogen at EOT in a subject who did not have a positive culture for that pathogen at baseline). Solid lines indicate a
significant difference from placebo in hierarchal testing; dashed lines indicate statistical significance was not reached. The $\alpha$ level for all end-points was 0.025 and the confidence interval is $97.5 \%$. 
TABLE 4 Treatment-emergent adverse events (TE-AEs) ${ }^{\#}$ (MedDRA classifications)

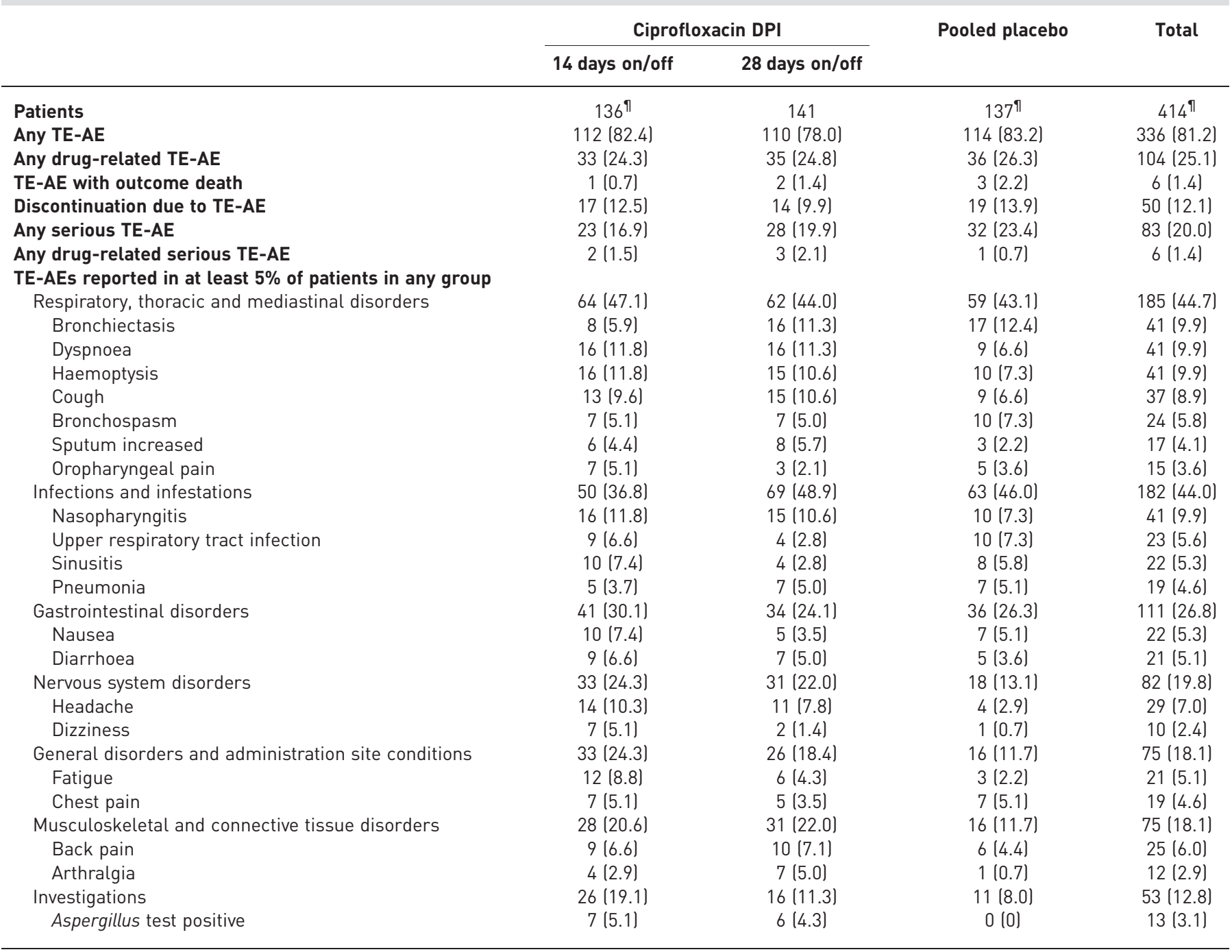

Data are presented as $\mathrm{n}$ or $\mathrm{n}(\%)$. MedDRA: Medical Dictionary for Regulatory Activities; DPI: dry powder for inhalation. ${ }^{\#}$ : defined as those that first occurred or worsened during the study from the first administration of study medication (ciprofloxacin DPI or placebo) until 30 days after administration of the last dose of study medication; ": two randomised subjects did not receive study medication lone in ciprofloxacin DPI 14 days on/off and one in placebo 28 days on/off).

was effective in patients with a range of different baseline characteristics, including patients with or without a positive baseline $P$. aeruginosa culture and those with or without chronic concomitant macrolide use. However, it should be noted that subgroup analyses were exploratory in nature and the small size of subgroups limits definitive conclusions. Post hoc calculations of the number needed to treat determined that 4.5 patients would need to be treated with ciprofloxacin DPI 14 days on/off to prevent one exacerbation over 336 days. Several secondary end-points (EMA/others analysis) were also statistically significant for the 14-day on/off arm, including a significant improvement in respiratory symptoms as measured by the SGRQ symptoms component score, which is noteworthy given the high symptom burden in bronchiectasis [13].

The results observed for ciprofloxacin DPI 28 days on/off were supportive of the efficacy seen in the ciprofloxacin DPI 14-day on/off arm, but did not reach the pre-defined significance level.

The patient enrolment criteria were designed to minimise variability in outcomes due to disease aetiology (e.g. excluding those with immune-deficiency disorders, active or actively treated nontuberculous mycobacterial lung infection, or active allergic bronchopulmonary aspergillosis) and to enrich the population with patients who were most likely to experience bacterial exacerbations. However, the mean number of exacerbations during the study was lower than the number of exacerbations in the previous 
year (as reported for study enrolment). Participation in a clinical trial is known to influence participant behaviour [14], and perhaps patients became more adherent to physiotherapy and ancillary treatments, thereby reducing fluctuations in baseline symptoms and potentially the number of exacerbations during the study. Data on ancillary treatments and adherence to such therapies were not, however, collected during the study and so we are unable to confirm this hypothesis.

Ciprofloxacin DPI had a good safety profile and was well tolerated; $>70 \%$ of patients completed treatment. Overall, treatment-emergent adverse event rates, including respiratory events, were generally low and comparable across treatment groups. No increased risk of serious systemic fluoroquinolone-associated events was observed. Potential systemic side-effects, such as nausea or headache, were infrequently reported (total $5.3 \%$ nausea and $7.0 \%$ headache) during this 48 -week study, probably reflecting the low systemic concentrations associated with ciprofloxacin DPI treatment [15].

As increases in MICs are a potential drawback of any antibiotic therapy, changes in the MICs of the seven pre-defined pathogens were studied. Use of a long-term inhaled antibiotic in chronic infection is expected to increase antimicrobial resistance by selecting for bacteria with resistance genes. The use of systemic breakpoints with inhaled therapies is, however, problematic and they are unlikely to accurately apply to this treatment scenario as concentrations achieved in sputum greatly exceed calculated MICs [15, 16]. Nonetheless, based on systemic breakpoints, $>20 \%$ of patients in each group had elevated MICs to ciprofloxacin at baseline, possibly reflecting the heavy burden of prior antibiotic use associated with frequent exacerbations in this patient population [17]. Subgroup analyses did not indicate reduced efficacy in patients who had baseline pathogens with MICs elevated above systemic breakpoints. Although $>50 \%$ of ciprofloxacin DPI-treated patients had at least one isolate with an elevated MIC at any time-point during the study, this rate fell to $<10 \%$ at EOS in patients who had susceptible pathogens at baseline (versus $2 \%$ in the placebo arm), which is similar to the resistance rate reported in a meta-analysis of inhaled antibiotic trials in patients with NCFB [18]. Increases in MIC may be offset by a net reduction in bacterial load and overall clinical benefits, including reduced exacerbations and therefore antibiotics. Additional studies will be required to monitor ciprofloxacin resistance during long-term use of ciprofloxacin DPI and to define relevant breakpoints for resistance to inhaled therapy.

We acknowledge the study has limitations. Varying definitions of exacerbation in clinical practice, a topic recently addressed by an expert panel [19], may have influenced patients' eligibility for this trial. There is currently considerable heterogeneity in how exacerbations are defined [19] and so it is likely that determination of exacerbations in the previous year varied among centres. Related to this, the definition of exacerbation used to ascertain eligibility for the trial relied on clinical judgement rather than specific criteria and was therefore less rigorous than that used for the primary end-points. Taken together, these factors may all have contributed to the lower-than-expected number of on-trial exacerbations. On the basis of this experience, we encourage investigators of future trials to utilise a rigorous definition for exacerbations qualifying as entry criteria. The potential drawback of this approach, however, is a further reduction in trial enrolment in an already limited population.

An additional methodological limitation was that computed tomography scans were not centrally validated and variation among centres may have affected assessment of disease severity or led to overestimation of radiological findings versus inclusion criteria. Validated prognostic indices for evaluating NCFB severity had not been published when the study was designed $[2,20]$ and the data collected in this trial did not encompass the complete information required to calculate severity scores, including radiological severity. Accordingly, the impact of therapy across disease severity groups could not be determined. Future studies may benefit from targeting patient populations with higher risks of exacerbation. The role of severity assessment tools has yet to be defined in clinical trials. The most appropriate method to measure change in health-related quality of life, particularly in clinical trials with investigational drugs, is also uncertain. Although RESPIRE 1 included both the SGRQ and Quality of Life-Bronchiectasis measures, these are based on different recall periods and the optimal time-point(s) for data collection with long-term cyclical therapy is unclear.

Finally, the 2:1 randomisation of active:placebo, while attractive to patients due to its higher probability of active treatment, meant that the smaller placebo arms were more susceptible to variances, which could potentially influence the outcomes of analyses between ciprofloxacin DPI and matching placebo groups.

In conclusion, the RESPIRE 1 trial has shown that therapy with ciprofloxacin DPI reduces exacerbations in patients with NCFB with frequent exacerbations and pathogens present in sputum. Statistically significant improvements were seen with 14 days on/off treatment, while trends toward improvement were seen with 28 days on/off treatment. Treatment was well tolerated. Consistent with other studies of long-term antibiotic therapy, increased MICs were seen. Our findings suggest that long-term intermittent 
inhaled antibiotic therapy with ciprofloxacin DPI may provide therapeutic benefit in selected patients with NCFB.

\section{Acknowledgements}

We thank the patients, investigators (see supplementary section S3) and study centres that contributed to RESPIRE

1. We also thank Ulrike Krahn (Bayer AG, Wuppertal, Germany) for assisting with statistical analysis, Jeff Alder (Bayer

US LLC, Whippany, NJ, USA) for microbiology data assessment and critically reviewing the data, Maxine Lau (Bayer

AG, Berlin, Germany) for pharmacovigilance input as well as Fusion MD (Montreal, Canada) and highfield: communication (Oxford, UK) for providing medical writing services with funding from Bayer AG. A. De Soyza, R. Wilson and J.S. Elborn acknowledge the support of EMBARC and the Medical Research Council-funded BRONCH-UK collaborative for peer support and advice in bronchiectasis.

\section{References}

1 Chalmers JD, Smith MP, McHugh BJ, et al. Short- and long-term antibiotic treatment reduces airway and systemic inflammation in non-cystic fibrosis bronchiectasis. Am J Respir Crit Care Med 2012; 186: 657-665.

2 Chalmers JD, Goeminne P, Aliberti S, et al. The Bronchiectasis Severity Index. An international derivation and validation study. Am J Respir Crit Care Med 2014; 189: 576-585.

3 Vendrell M, de Gracia J, Olveira C, et al. Diagnostico y tratamiento de las bronquiectasias. SEPAR. [Diagnosis and treatment of bronchiectasis. Spanish Society of Pneumology and Thoracic Surgery.] Arch Bronconeumol 2008; 44: $629-640$.

4 Chang AB, Bell SC, Byrnes CA, et al. Chronic suppurative lung disease and bronchiectasis in children and adults in Australia and New Zealand. Med J Aust 2010; 193: 356-365.

5 Pasteur MC, Bilton D, Hill AT, et al. British Thoracic Society guideline for non-CF bronchiectasis. Thorax 2010 65: Suppl. 1, 1-58

6 Weers J, Tarara T. The PulmoSphere ${ }^{\mathrm{TM}}$ platform for pulmonary drug delivery. Ther Deliv 2014; 5: 277-295.

7 Stass H, Nagelschmitz J, Kappeler D, et al. Ciprofloxacin dry powder for inhalation in patients with noncystic fibrosis bronchiectasis or chronic obstructive pulmonary disease, and in healthy volunteers. J Aerosol Med Pulm Drug Deliv 2017; 30: 53-63.

8 Wilson R, Welte T, Polverino E, et al. Ciprofloxacin dry powder for inhalation in non-cystic fibrosis bronchiectasis: a phase II randomised study. Eur Respir J 2013; 41: 1107-1115.

9 Aksamit $\mathrm{T}$, Bandel TJ, Criollo $\mathrm{M}$, et al. The RESPIRE trials: two phase III, randomised, multicentre, placebo-controlled trials of Ciprofloxacin Dry Powder for Inhalation (Ciprofloxacin DPI) in non-cystic fibrosis bronchiectasis. Contemp Clin Trials 2017; 58: 78-85.

10 Lo D, VanDevanter DR, Flume P, et al. Aerosolized antibiotic therapy for chronic cystic fibrosis airway infections: continuous or intermittent? Respir Med 2011; 105: Suppl. 2, S9-S17.

11 Barker AF, O’Donnell AE, Flume P, et al. Aztreonam for inhalation solution in patients with non-cystic fibrosis bronchiectasis (AIR-BX1 and AIR-BX2): two randomised, double-blind, placebo-controlled phase 3 trials. Lancet Respir Med 2014; 2: 738-749.

12 Haworth CS, Wanner A, Froehlich J, et al. Inhaled liposomal ciprofloxacin in patients with bronchiectasis and chronic Pseudomonas aeruginosa infection: results from two parallel phase III trials (ORBIT-3 and -4)). Multidiscip Respir Med 2018; in press.

13 Wilson CB, Jones PW, O'Leary CJ, et al. Validation of the St. George's Respiratory Questionnaire in bronchiectasis. Am J Respir Crit Care Med 1997; 156: 536-541.

14 McCambridge J, Witton J, Elbourne DR. Systematic review of the Hawthorne effect: new concepts are needed to study research participation effects. J Clin Epidemiol 2014; 67: 267-277.

15 Stass H, Delesen H, Nagelschmitz J, et al. Safety and pharmacokinetics of ciprofloxacin dry powder for inhalation in cystic fibrosis: a phase I, randomized, single-dose, dose-escalation study. J Aerosol Med Pulm Drug Deliv 2015; 28: $106-115$

16 Flume PA. A role for aerosolized antibiotics. Pediatr Pulmonol 2008; 43: S29-S34.

17 de la Rosa D, Martinez-Garcia MA, Olveira C, et al. Annual direct medical costs of bronchiectasis treatment: impact of severity, exacerbations, chronic bronchial colonization and chronic obstructive pulmonary disease coexistence. Chron Respir Dis 2016; 13: 361-371.

18 Brodt AM, Stovold E, Zhang L. Inhaled antibiotics for stable non-cystic fibrosis bronchiectasis: a systematic review. Eur Respir J 2014; 44: 382-393.

19 Hill AT, Haworth CS, Aliberti S, et al. Pulmonary exacerbation in adults with bronchiectasis: a consensus definition for clinical research. Eur Respir J 2017; 49: 1700051.

20 Martinez-Garcia MA, de Gracia J, Vendrell Relat MV, et al. Multidimensional approach to non-cystic fibrosis bronchiectasis: the FACED score. Eur Respir J 2014; 43: 1357-1367. 